\title{
Clinical characteristics, outcome and early induction deaths in patients with acute promyelocytic leukaemia: a five-year experience at a tertiary care centre
}

\author{
Farheen $\underline{\text { Karim }}^{1}$, MBBS, FCPS, Usman Shaikh${ }^{1}$, MBBS, FCPS, Salman Naseem $\underline{\text { Adil }}^{1}$, MBBS, FCPS,
} Mohammad Khurshid ${ }^{1}$, MBBS, FRCPath

\begin{abstract}
INTRODUCTION Acute promyelocytic leukaemia (APL) is a distinct clinical and biological subtype of acute myeloid leukaemia. APL is notorious for causing early death during induction therapy, resulting in induction failure. The aim of our study was to report the clinical characteristics, outcome and early induction deaths with regard to patients with APL seen at our hospital.

METHODS This was a retrospective study carried out at Aga Khan University Hospital, Karachi, Pakistan. Patients aged $>15$ years diagnosed with APL within the period September 2007-September 2012 were included in the study.

RESULTS Within the study period, 26 patients were diagnosed with APL based on morphology and the detection of $\mathrm{t}(15 ; 17)$ (q24.1;q21.1) and promyelocytic leukaemia-retinoic acid receptor alpha (PML-RARA). The male to female ratio was 1:1. The median age of the patients was 41 (range 16-72) years. In all, there were $13(50.0 \%)$ high-risk patients, and early induction death rate was $61.5 \%$. Causes of early induction deaths $(n=16)$ included haemorrhage in $7(43.8 \%)$ patients, differentiation (ATRA) syndrome in 7 (43.8\%) and infection in 2 (12.5\%). The survival rate among patients who survived the early period was $70 \%$ at 42 months. The relapse rate was $30 \%$.

CONCLUSION Early induction death rate was very high in patients with APL. The most common cause of early induction death in our study was haemorrhage. Outcome among patients with APL was found to be better among those who survived the initial period.
\end{abstract}

Keywords: adults, death, early, leukaemia, promyelocytic

\section{INTRODUCTION}

A distinct clinical and biological subtype of acute myeloid leukaemia (AML), acute promyelocytic leukaemia (APL) was previously classified as AML-M3 in the older French-AmericanBritish (FAB) classification system, ${ }^{(1,2)}$ and then as APL with $t(15 ; 17)(q 24.1 ; q 21.1)$ and promyelocytic leukaemia-retinoic acid receptor alpha (PML-RARA) by World Health Organization. ${ }^{(3)}$ APL makes up nearly $5 \%-8 \%$ of cases of AML. ${ }^{(4)}$ The incidence of APL increases during the teenage years and reaches a plateau in early adulthood. It is uncommon in children aged less than ten years and among elderly people over 60 years old. ${ }^{(5)} \mathrm{APL}$ is characterised by the predominance of abnormal promyelocytes in the bone marrow and a specific chromosomal translocation $t(15 ; 17)(q 24.1 ; q 21.1)$ - resulting in a fusion transcript between the promyelocytic (PML) gene found on chromosome 15 and the retinoic acid receptor alpha (RARA) gene present on chromosome 17 (i.e. PML-RARA). ${ }^{(6)}$

There are two morphological variants of $A P L$, with the most common being the hypergranular variant, in which promyelocytes are populated with abundant azurophilic granules and may contain multiple Auer rods, which is a classical finding. ${ }^{(4)}$ Accounting for about $25 \%$ of patients with $\mathrm{APL}$, the less common microgranular variant of APL (M3v) is more aggressive than the hypergranular variant, and on light microscopy is seen to be morphologically characterised by immature myeloid cells that lack azurophilic granules and have convoluted nuclei. ${ }^{(7)}$

APL accounted for high mortality rates during induction therapy because of bleeding diathesis in the pre-all trans retinoic acid (ATRA) era.$^{(8)}$ However, the late-1980s discovery of the clinical efficacy of ATRA, which promotes the terminal differentiation of malignant promyelocytes to mature neutrophils, changed the natural history of APL. ${ }^{(9,10)}$ Regimens using a combination of ATRA and anthracyclines (such as idarubicin or daunorubicin) have been shown to achieve very high remission rates of up to $90 \%$ and prolonged event-free survival in patients with APL. ${ }^{(1-13)}$ The simultaneous use of ATRA and anthracyclines has now become the standard treatment for induction and consolidation in APL, and it is possible to cure most patients using these agents. ${ }^{(14)}$ Around $80 \%-90 \%$ of patients with APL are cured of the disease, if they survive induction to achieve complete remission. ${ }^{(15)}$

While death during the induction phase from causes such as haemorrhage, differentiation syndrome (DS) and infection poses a significant challenge in early treatment, resistance to therapy is an uncommon cause of induction failure. ${ }^{(16)} \mathrm{APL}$ is a notorious cause of early haemorrhagic deaths, and this has been recognised as early as 1957. ${ }^{(17)}$ The microgranular variant of APL is associated with a higher risk of early haemorrhagic death. ${ }^{(18,19)}$ Patients can have severe bleeding diathesis with widespread petechial rash, central nervous system (CNS) bleeding, gastrointestinal

${ }_{1}^{1}$ Department of Pathology and Microbiology, Aga Khan University Hospital, Karachi, Pakistan

Correspondence: DrFarheen Karim, Instructor, Section of Hematology, Department of Pathology and Microbiology, Aga Khan University Hospital, Stadium Road, Karachi 74800 , Pakistan. farheen.mahar@aku.edu 
haemorrhages, pulmonary haemorrhages and excessive loss of blood from sites of trauma. ${ }^{(18,19)}$ The risk factors associated with haemorrhagic death during induction in the ATRA era include peripheral blast count over $30 \%$, abnormal serum creatinine levels and the presence of coagulopathy. ${ }^{(20)}$ DS, also known as retinoic acid syndrome, is another common cause of early death, resulting from the use of differentiating agents (such as ATRA or arsenic trioxide) in patients with APL during the induction phase. Frequently observed at the onset of DS, peak white blood cell (WBC) count is predictive of DS. ${ }^{(20)}$

Early death is a major cause of induction failure, accounting for treatment failures in about $25 \%$ of patients with APL. ${ }^{(21)}$ According to the Programa Español de Tratamientos en Hematología (PETHEMA) group, factors predictive of death during induction included $\mathrm{WBC}>10 \times 10^{9} / \mathrm{L}$, age over 60 years, creatinine level $>1.4 \mathrm{mg} / \mathrm{dL}$ and male gender. ${ }^{(21)}$ However, population-based studies suggest that the frequency of early deaths during the induction phase has not changed much in recent years. ${ }^{(22,23)}$ Given the risk of early death and fatal bleeding, APL must be considered a medical emergency requiring early recognition and prompt initiation of treatment at first suspicion, without waiting for confirmatory cytogenetic or molecular genetic diagnosis.

Few studies have reported the incidence of induction failure due to early death and the risk factors associated with it among patients with APL. We herein present the clinical characteristics, outcome and early induction deaths in patients with APL at our hospital.

\section{METHODS}

This was a retrospective descriptive study conducted at Aga Khan University Hospital, Karachi, Pakistan, which is a 700-bed tertiary care academic hospital. We collected the data of all adult patients with morphologically, cytogenetically and molecularly confirmed diagnoses of APL admitted to the hospital between September 2007 and September 2012. The medical records of all patients were reviewed in detail to gather information regarding clinical characteristics, chemotherapeutic protocols used, outcome, and causes of early induction deaths. Laboratory data of the patients, including complete blood count, peripheral blood and bone marrow morphologies, cytogenetics for $t(15 ; 17)$ (q24.1; 21.1$)$ and PML-RARA, and coagulation profile results, were collected using the computerised Integrated Laboratory Management Services system, which maintains a record of the laboratory results of all patients.

Patients were categorised into three groups (i.e. low risk, intermediate risk and high risk), according to the Gruppo Italiano Malattie Ematologiche dell'Adulto (GIMEMA) and PETHEMA studies. ${ }^{(24,25)}$ Low-risk patients had leucocyte counts $\leq 10 \times$ $10^{9} / \mathrm{L}$ and platelets $>40 \times 10^{9} / \mathrm{L}$, intermediate-risk patients had leucocytes $\leq 10 \times 10^{9} / \mathrm{L}$ and platelets $<40 \times 10^{9} / \mathrm{L}$, and high-risk patients had leucocytes $>10 \times 10^{9} / \mathrm{L}$.

Patients were treated according to the PETHEMA LPA 99 protocol. ${ }^{(24)}$ Induction therapy, i.e. all-trans retinoic acid plus idarubicin (AIDA) therapy, involved the administration of ATRA $\left(45 \mathrm{mg} / \mathrm{m}^{2}\right.$ per day in two divided doses until complete response is achieved) and idarubicin (12 mg/ $\mathrm{m}^{2}$ intravenous push on Days 2, 4, 6 and 8). Consolidation therapy consisted of three cycles of anthracycline-based therapy (idarubicin $5-7 \mathrm{mg} / \mathrm{m}^{2} \times 4$ days; mitoxantrone $10 \mathrm{mg} / \mathrm{m}^{2} \times 5$ days; and idarubicin $12 \mathrm{mg} / \mathrm{m}^{2} \times$ 1-2 days), and also including a 15-day period of ATRA during consolidation cycles. This was followed by two years of maintenance therapy with daily oral 6-mercaptopurine, weekly oral methotrexate and intermittent ATRA $\left(45 \mathrm{mg} / \mathrm{m}^{2} \times 15\right.$ days every three months) following diagnosis. ${ }^{(24,26)}$ In some patients, idarubicin was replaced by daunorubicin, depending on the physician's preference.

DS diagnosis was made based on clinical and radiological features in the context of induction therapy with ATRA, in the presence of at least two of the following signs and symptoms: (a) unexplained fever; (b) weight gain > $5 \mathrm{~kg}$; (c) unexplained hypotension; (d) acute renal failure; and (e) acute respiratory distress with chest radiographs showing the presence of pulmonary infiltrates or pleuropericardial effusion. ${ }^{(27)}$ In our study, early death was defined as death from any cause during the period between the first day of hospitalisation and the 30th day from the initiation of therapy. ${ }^{(27)}$

Transfusion thresholds included platelet transfusion at platelet count $<30 \times 10^{9} / \mathrm{L}$ and packed red cell transfusion at haemoglobin $<9 \mathrm{~g} / \mathrm{dL}$. Cryoprecipitates were transfused if fibrinogen was $<150 \mathrm{mg} / \mathrm{dL}$, and fresh frozen plasma was given if prothrombin time and activated partial thromboplastin time were 1.5 times of their respective normal upper limits. All neutropenic patients were treated with empiric broad-spectrum antibiotics according to the hospital's protocol.

Data was analysed using the Statistical Package for the Social Sciences version 19.0 (SPSS Inc, Chicago, IL, USA). Overall survival curves were calculated using the Kaplan-Meier method. Patient characteristics were compared using chi-square analysis. Approval from the institutional ethical review committee for the study was not obtained, as such approval is not required for the analysis of retrospective data, provided that anonymity is maintained during analysis.

\section{RESULTS}

In our study, 26 patients were diagnosed with APL based on morphology and the detection of $t(15 ; 17)(q 24.1 ; q 21.1)$ and PML-RARA mutation during the study period. The $t(15 ; 17)$ (q24.1; q21.1) translocation was detected in 19 (73.1\%) patients. However, in 7 (26.9\%) patients where the translocation could not be detected using conventional cytogenetic techniques, the diagnosis was confirmed by the presence of PML-RARA mutation via fluorescence in situ hybridisation. On morphological examination, all 26 (100.0\%) patients had classical hypergranular $\mathrm{APL}$, and the microgranular variant was not found in any patient. All patients were diagnosed with de novo APL.

There were $13(50.0 \%)$ men and $13(50.0 \%)$ women in our study group, with a male to female ratio of $1: 1$. The median age of patients was 41 (range 16-72) years. The baseline laboratory tests of all patients diagnosed with APL are summarised in Table I. When stratified according to risk, there were $6(23.1 \%)$ patients 
Table I. Baseline laboratory parameters.

\begin{tabular}{lc}
\hline Parameter & Mean (range) \\
\hline Haemoglobin $(\mathrm{g} / \mathrm{dL})$ & $8.5(4.2-11.5)$ \\
White blood cells $\left(\times 10^{9} / \mathrm{L}\right)$ & $28.1(0.3-14.9)$ \\
Platelets ( $\left.\times 10^{9} / \mathrm{L}\right)$ & $40(8-227)$ \\
Prothrombin time (s) & $14.2(11.4-21)$ \\
Activated partial thromboplastin time (s) & $28(21-120)$ \\
Fibrinogen (mg/dL) & $86(10-300)$ \\
D-dimer (mg/L FEU) & $13.1(0-0.8)$ \\
\hline
\end{tabular}

FEU: fibrinogen equivalent units

in the low risk group, 7 (26.9\%) in the intermediate risk group and $13(50.0 \%)$ patients in the high risk group. DS was observed in $7(26.9 \%)$ patients, and was fatal in all.

For induction, 20 patients received a combination of ATRA and an anthracycline; the choice of anthracycline was daunorubicin in 9 (45.0\%) patients and idarubicin in 11 (55.0\%) patients. The remaining six patients in our study received only ATRA, as they were not fit for chemotherapy.

Among the 26 patients in the study, 19 (73.1\%) died, while $7(26.9 \%)$ were alive and in remission on follow-up at 42 months. Overall survival rate, stratified according to risk group, showed that low-risk patients had a survival rate of $50.0 \%$ at 42 months, while that in intermediate-risk patients was $28.6 \%$. Prognosis was worst at 42 months for patients in the high-risk group, with a survival rate of $15.4 \%$. Based on the choice of anthracyclines, no statistically significant difference was observed in patient outcome between patients who received daunorubicin and those who received idarubicin. Overall survival and survival stratified according to risk group in our patients with APL using Kaplan-Meier curves are depicted in Figs. 1 and 2, respectively.

In our study cohort $(\mathrm{n}=26)$, early induction death was seen in $16(61.5 \%)$ patients with APL. The causes of early induction deaths were haemorrhage $(n=7,43.8 \%)$, infection $(n=2$, $12.5 \%)$ and DS (ATRA) $(n=7,43.8 \%)$. In all $(n=26), 10(38.5 \%)$ patients completed the treatment protocol, including maintenance therapy. However, 3 (30.0\%) out of 10 patients relapsed after a median duration of 730 days after achieving complete remission, with bone marrow relapse in two patients, and both bone marrow and CNS relapses in the other. Among these three patients, one patient each belonged to the low-risk, intermediate-risk and highrisk groups. All patients who relapsed were given idarubicin and ATRA during induction. All three patients died following relapse. The survival rate among patients who survived the early period was $70 \%$ at 42 months.

\section{DISCUSSION}

Previously considered one of the most fatal subtypes of AML due to the bleeding diathesis seen in patients, APL has now become the most curable form of AML. ${ }^{(28)}$ This tremendous change in the outcome of APL became possible with the advent of ATRA; various clinical trials have shown complete remission in $90 \%-95 \%$ of patients with APL when ATRA was given in combination with an anthracycline. ${ }^{(28-30)}$ Based on these trials,

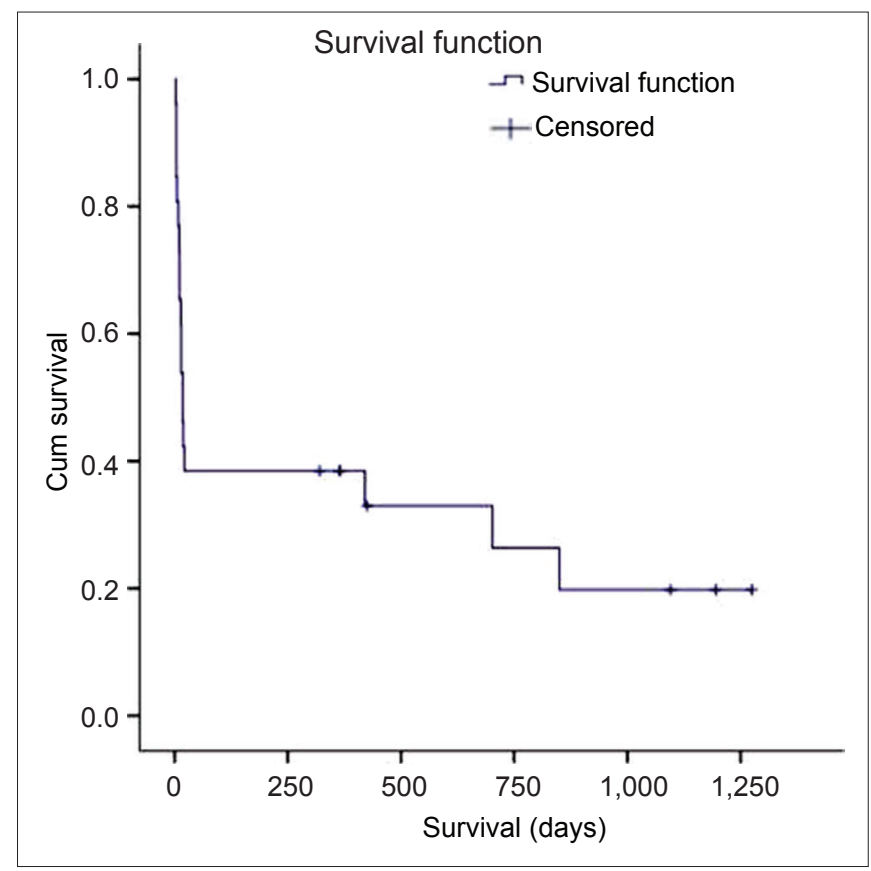

Fig. 1 Graph shows the overall survival of patients with acute promyelocytic leukaemia.

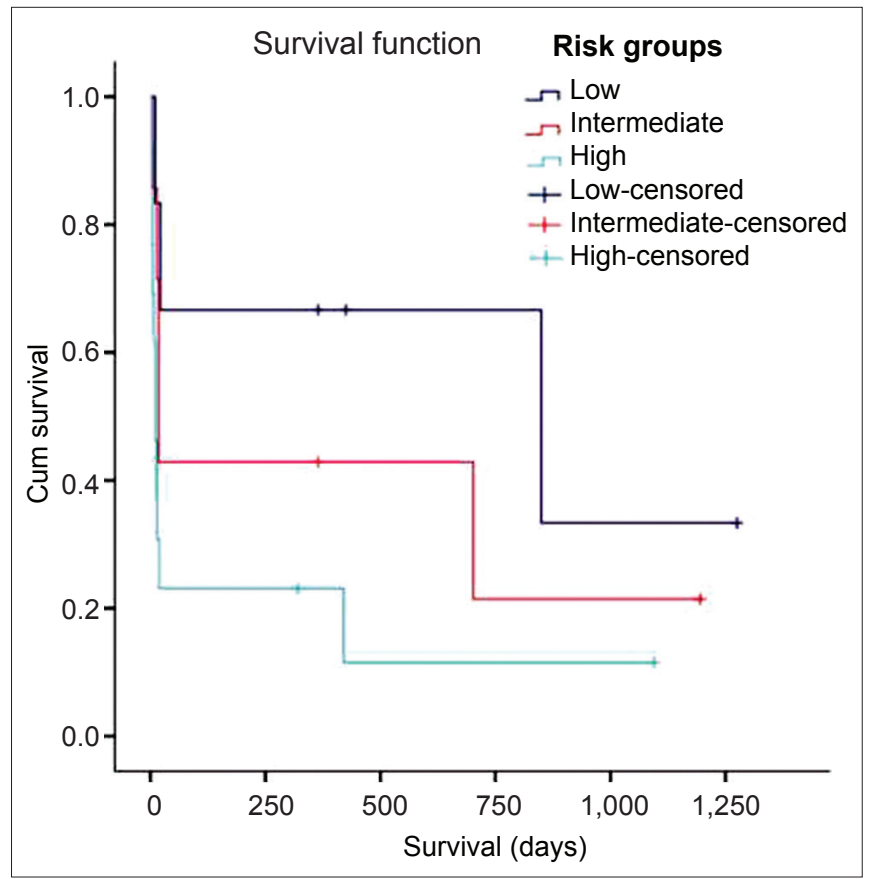

Fig. 2 Graph shows the survival of patients with acute promyelocytic leukaemia, stratified according to level of risk.

ATRA and anthracycline-based regimens have become standard treatment for APL. ${ }^{(31,32)}$ However, despite improvement in the survival of patients with APL with modern therapy, induction failure due to early induction deaths remains a major challenge in APL treatment. Haemorrhage causes early death in 5\%-10\% of patients with APL in developed countries, and in around $20 \%-30 \%$ of patients in developing countries. ${ }^{(20)}$ The common causes of induction deaths in APL patients include haemorrhage, infection and DS. ${ }^{(20)}$

The majority $(50.0 \%)$ of patients with APL in our study belonged to the high-risk category, with WBC > $10 \times 10^{9} / \mathrm{L}$ at 
the time of diagnosis. In contrast, only approximately $22 \%$ of patients were in the high-risk groups in the larger GIMEMA and PETHEMA trials. ${ }^{(24,25)}$

Early death rates among patients with APL reported in the literature vary widely. While early induction death was seen in $61.5 \%$ of our patients, another study ${ }^{(33)}$ from the region reported a mortality rate of $28.6 \%$ in patients with APL who were administered anthracycline and ATRA therapy. While a Turkish study reported a high incidence of early induction death at $40 \%$, ${ }^{(20)}$ a high early death rate of $32 \%$ was reported in a Brazilian study, ${ }^{(29)}$ and a Greek study found early death rate to be $14.9 \% .{ }^{(34)}$ In another series, the early death rate among patients with APL was $21.5 \%$. ${ }^{(35)}$ These population-based studies suggest that the early death rates depicted in clinical trials do not correspond to real-life circumstances and have not changed much in the ATRA era, as seen in our study.

In our study, the causes of early induction death were haemorrhage $(n=7,43.8 \%)$, infection $(n=2,12.5 \%)$ and DS $(\mathrm{n}=7,43.8 \%$ ). de la Serna et al reported that the most common cause of early induction death was haemorrhage (5\%) followed by infection (2.3\%) and DS (1.4\%) in APL patients on the AIDA regimen. ${ }^{(19)}$ In our study, the most common type of haemorrhage observed was intracranial bleeding, which was observed in $5(71.4 \%)$ patients and confirmed via imaging studies. In the other two patients with haemorrhage, one had suspected intracranial bleeding, which could not be confirmed by imaging studies, while the site of haemorrhage was not documented in the other. All our patients with haemorrhage had low platelet counts, with a mean platelet count of $19 \times 10^{9} / \mathrm{L}$ and a mean fibrinogen level of $213 \mathrm{mg} / \mathrm{dL}$. Although we maintained target fibrinogen level in all patients, we failed to maintain target platelet count $>30$ $\times 10^{9} / \mathrm{L}$ in some patients despite aggressive supportive therapy. The reason for low platelet counts in these patients was platelet refractoriness. Failure to maintain the target platelet count may have been a factor contributing to haemorrhage in these patients. It was also observed that some patients had intracranial bleeding despite maintaining platelet count $>30 \times 10^{9} / \mathrm{L}$. Our findings suggest that due to the high risk of bleeding diathesis in patients with $\mathrm{APL}$, clinicians should aim for higher platelet count of around $50 \times 10^{9} / \mathrm{L}$ during induction therapy, which may help to prevent death from haemorrhage in these patients.

Among the two patients in our study with early induction death due to infection, one had Gram-negative septicaemia from Pseudomonas aeruginosa and Klebsiella spp., along with Aspergillus fungal infection, and the other had Gram-positive septicaemia due to Staphylococcus spp. Both patients were neutropenic at the time of infection, with a mean absolute neutrophil count of $1 \times 10^{9} / \mathrm{L}$. However, both patients died despite prompt administration of intravenous antibiotics and antifungal agents.

Patients in our cohort who survived induction therapy showed very good response, with an overall survival rate of $70 \%$ at 42 months. The incidence of DS in our study was $26.9 \%$, which is comparable to the overall incidence of DS reported in the LPA 99 trial $(24.8 \%)^{(21)}$ and by Sanz et al in 2010 (28\%). ${ }^{(36)}$ However, mortality associated with DS in these earlier trials ranged from $1 \%$ to $14 \%$. In contrast, DS in our study was associated with a mortality rate of $100 \%$. It should be noted that prophylaxis for DS was not provided earlier in our set-up, as the effectiveness of the regimen could not be established based on previous reports. However, in view of the high mortality associated with DS in our patients, prophylaxis for DS using dexamethasone is now incorporated in the treatment regimen of patients with APL at our centre, with every patient started on ATRA now being given $10 \mathrm{mg}$ of prophylactic dexamethasone daily. Morbidity and mortality from DS have been significantly reduced in our patients with APL since prophylaxis with dexamethasone was begun. These findings will need to be confirmed in future studies. A relapse rate of $30 \%$ was observed in our patients, which is in concordance with relapse rates ranging from $20 \%$ to $30 \%$ reported in other studies, despite consolidation and maintenance therapy. ${ }^{(6,11)}$

In sharp contrast to the projected survival of patients with APL in most international studies, ${ }^{(31,32)}$ the very low survival rate seen among our patients with APL at 42 months was probably related to the very high early death rates observed in our study. The worst outcome was observed in high-risk patients, with a survival of only $15.4 \%$ at 42 months. The majority of our patients (50.0\%) were in the high-risk category. Prognosis among patients in the highrisk group is typically expected to be bad. Similar results were reported by Serefhanoglu et al, who found the overall 2.5-year survival of high-risk patients to be $33.6 \%$. ${ }^{(20)}$ A higher percentage of patients in the study by Serefhanoglu et $\mathrm{al}^{(20)}$ belonged to the high-risk category (34\%), similar to our study.

Our study had its share of limitations. Firstly, late presentation was a problem encountered in our cohort. In developing countries such as ours, patients tend to first be treated by local physicians, and are usually referred quite late to tertiary-care centres, which results in delayed diagnosis and initiation of therapy. Furthermore, many of our patients only presented to our centre after being treated at the primary care setting. Some of our patients who showed a worsening clinical condition were not deemed fit to receive chemotherapy and died soon after admission. Secondly, FMS-like tyrosine kinase 3 (FLT3)/internal tandem duplication (ITD) mutations are known to be associated with poor outcome in patients with APL. ${ }^{(37)}$ However, we did not ascertain the FLT-3 mutation status of our patients in the present study, as this facility was not available at our centre then. We aim to include in future studies investigations on FLT-3 mutation to observe its effect on the outcome of patients with APL.

Aggressive supportive treatment during the induction phase, maintenance higher platelet transfusion thresholds and administration of DS prophylaxis were some of the steps taken to mitigate the high mortality rates of patients with APL in our study. Creating awareness among general practitioners by conducting educational programmes, lectures and workshops on the importance of early diagnosis and quick referrals to specialised centres for the treatment of APL could be significant in helping to reduce the early mortality rate associated with late referral in these patients. Future studies should assess the impact of such interventions on the outcome of APL. 
In conclusion, early induction death rate was very high in patients with APL. The most common cause of early induction death in our study was haemorrhage. We also found that the outcome of APL was better, provided patients survived the initial treatment period. Early diagnosis and prompt treatment with standard chemotherapy protocols and supportive management is therefore essential in patients with APL.

\section{REFERENCES}

1. Walter RB, Othus M, Burnett AK, et al. Significance of FAB subclassification of "acute myeloid leukemia, NOS" in the 2008 WHO classification: analysis of 5848 newly diagnosed patients. Blood 2003; 121:2424-31.

2. Bennett JM, Catovsky D, Daniel MT, et al. Proposed revised criteria for the classification of acute myeloid leukemia. A report of the French-AmericanBritish Cooperative Group. Ann Intern Med 1985 Oct; 103:620-5.

3. Swerdlow $\mathrm{SH}$, Campo $\mathrm{E}$, Harris $\mathrm{NL}$, et al, eds. WHO Classification of Tumours of Haematopoietic and Lymphoid Tissues. 4th ed. Lyon: IARC Press, 2008.

4. Stanley M, McKenna RW, Ellinger G, Brunning RD. Classification of 358 Cases of Acute Myeloid Leukemia by FAB Criteria: Analysis of Clinical and Morphologic Features. In: Bloomfield CD, ed. Chronic and Acute Leukemias in Adults. Boston: Martinus Nijhoff Publishers, 1985: 147-74.

5. Sanz MA, Grimwade D, Tallman MS, et al. Management of acute promyelocytic leukemia: recommendations from an expert panel on behalf of the European LeukemiaNet. Blood 2009; 113:1875-91.

6. Candoni A, Damiani D, Michelutti A, et al. Clinical characteristics, prognostic factors and multidrug-resistance related protein expression in 36 adult patients with acute promyelocytic leukemia. Eur J Haematol 2003; 71:1-8.

7. Golomb HM, Rowley JD, Vardiman JW, Testa HR, Butler A. "Microgranular" acute promyelocytic leukemia: a distinct clinical, ultrastructural, and cytogenetic entity. Blood 1980; 55:253-9.

8. Huang ME, Ye YC, Chen SR, et al. Use of all-trans retinoic acid in the treatment of acute promyelocytic leukemia. Blood 1988; 72:567-72.

9. Zhang L, Zhu X. Epidemiology, diagnosis and treatment of acute promyelocytic leukemia in children: the experience in China. Mediterr J Hematol Infect Dis 2012; 4:e2012012.

10. Tallman MS, Nabhan C. Management of acute promyelocytic leukemia. Curr Oncol Rep 2002; 4:381-9.

11. Douer D. Advances in the treatment of relapsed acute promyelocytic leukemia. Acta Haematol 2002; 107:1-17.

12. Tallman MS, Nabhan C, Feusner JH, Rowe JM. Acute promyelocytic leukemia: evolving therapeutic strategies. Blood 2002; 99:759-67.

13. Sanz MA, Martín G, Lo Coco F. Choice of chemotherapy in induction, consolidation and maintenance in acute promyelocytic leukemia. Best Pract Res Clin Haematol 2003; 16:433-51.

14. Tallman MS, Altman JK. How I treat acute promyelocytic leukemia. Blood 2009; 114:5126-35.

15. Di Bona E, Avvisati G, Castaman G, et al. Early haemorrhagic morbidity and mortality during remission induction with or without all-trans retinoic acid in acute promyelocytic leukemia. Br J Haematol 2000; 108:689-95.

16. Hillestad LK. Acute promyelocytic leukemia. Acta Med Scand 1957; 159:189-94.

17. Rovelli A, Biondi A, Cantù Rajnoldi A, et al. Microgranular variant of acute promyelocytic leukemia in children. J Clin Oncol 1992; 10:1413-8.

18. Tallman MS, Brenner B, Serna Jde L, et al. Meeting report. Acute promyelocytic leukemia-associated coagulopathy, 21 January 2004, London, United Kingdom. Leuk Res 2005; 29:347-51.

19. de la Serna J, Montesinos P, Vellenga E, et al. Causes and prognostic factors of remission induction failure in patients with acute promyelocytic leukemia treated with all-trans retinoic acid and idarubicin. Blood 2008; 111:3395-402.
20. Serefhanoglu S, Buyukasik Y, Goker H, et al. Clinical features and outcomes of 49 Turkish patients with acute promyelocytic leukemia who received ATRA and anthracyclines (PETHEMA protocol) therapy. Leuk Res 2010; 34:e317-9.

21. Sanz MA, Martín G, González M, et al. Risk-adapted treatment of acute promyelocytic leukemia with all-trans-retinoic acid and anthracycline monochemotherapy: a multicenter study of the PETHEMA group. Blood 2004; 103:1237-43.

22. Lehmann S, Ravn A, Carlsson L, et al. Continuing high early death rate in acute promyelocytic leukemia: a population-based report from the Swedish Adult Acute Leukemia Registry. Leukemia 2011; 25:1128-34.

23. Park JH, Qiao B, Panageas KS, Schymura MJ, Jurcic JG, Rosenblat TL, et al. Early death rate in acute promyelocytic leukemia remains high despite all-trans retinoic acid. Blood 2011: 118:1248-54.

24. Esteve J, Escoda L, Martín G, et al. Outcome of patients with acute promyelocytic leukemia failing to front-line treatment with all-trans retinoic acid and anthracycline-based chemotherapy (PETHEMA protocols LPA96 and LPA99): benefit of an early intervention. Leukemia 2007; 21:446-52.

25. Avvisati G, Lo Coco F, Diverio D, et al. AIDA (all-trans retinoic acid + idarubicin) in newly diagnosed acute promyelocytic leukemia: a Gruppo Italiano Malattie Ematologiche Maligne dell'Adulto (GIMEMA) pilot study. Blood 1996; 88:1390-8.

26. Avvisati G, Lo-Coco F, Paoloni FP, et al. AIDA 0493 protocol for newly diagnosed acute promyelocytic leukemia: very long-term results and role of maintenance. Blood 2011; 117:4716-25.

27. Montesinos P, Bergua JM, Vellenga $E$, et al. Differentiation syndrome in patients with acute promyelocytic leukemia treated with all-trans retinoic acid and anthracycline chemotherapy: characteristics, outcome, and prognostic factors. Blood 2009; 113:775-83.

28. Wang ZY, Chen Z. Acute promyelocytic leukemia: from highly fatal to highly curable. Blood 2008; 111:2505-15.

29. Tallman MS, Andersen JW, Schiffer CA, et al. All-trans retinoic acid in acute promyelocytic leukemia: long-term outcome and prognostic factor analysis from the North American Intergroup protocol. Blood 2002; 100:4298-302.

30. Jacomo RH, Melo RA, Souto RF, et al. Clinical features and outcomes of 134 Brazilians with acute promyelocytic leukemia who received ATRA and anthracyclines. Haematologica 2007; 92:1431-2.

31. Mandelli F, Diverio D, Avvisati G, et al. Molecular remission in PML/ RAR alpha-positive acute promyelocytic leukemia by combined all-trans retinoic acid and idarubicin (AIDA) therapy. Gruppo Italiano-Malattie Ematologiche Maligne dell'Adulto and Associazione Italiana di Ematologia ed Oncologia Pediatrica Cooperative Groups. Blood 1997; 90:1014-21.

32. Kanamaru A, Takemoto $Y$, Tanimoto M, et al. All-trans retinoic acid for the treatment of newly diagnosed acute promyelocytic leukemia. Japan Adult Leukemia Study Group. Blood 1995; 85:1202-6.

33. Raza S, Ullah K, Ahmed P, Khan B. Comparison of anthracycline-based combination chemotherapy with or without all-trans retinoic acid in acute promyelocytic leukemia. J Coll Physicians Surg Pak 2008; 18:546-50.

34. Pagoni M, Garofalaki M, Panitsas F, et al. Acute promyelocytic leukemia: an experience on 95 greek patients treated in the all-trans-retinoic Acid era. Mediterr J Hematol Infect Dis 2011; 3:e2011053.

35. Mitrovic M, Suvajdzic N, Bogdanovic A, et al. International Society of Thrombosis and Hemostasis Scoring System for disseminated intravascular coagulation $\geq 6$ : a new predictor of hemorrhagic early death in acute promyelocytic leukemia. Med Oncol 2013; 30:478.

36. Sanz MA, Montesinos P, Rayón C, et al. Risk-adapted treatment of acute promyelocytic leukemia based on all-trans retinoic acid and anthracycline with addition of cytarabine in consolidation therapy for high-risk patients: further improvements in treatment outcome. Blood 2010; 115:5137-46.

37. Au WY, Fung A, Chim CS, et al. FLT-3 aberrations in acute promyelocytic leukaemia: clinicopathological associations and prognostic impact. $\mathrm{Br}$ J Haematol 2004; 125:463-9. 